\title{
Excitation of Coherent Spin Waves in GdFeCo film
}

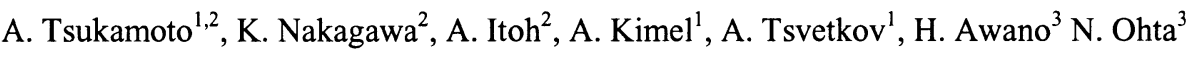 \\ A. Kirilyuk ${ }^{1}$, and Th. Rasing ${ }^{1}$ \\ ${ }^{1}$ NSRIM Institute, University of Nijmegen, Toernooiveld 1, 6525 ED Nijmegen, the Netherlands \\ ${ }^{2}$ College of science and technology, Nihon University, 7-24-1 Funabashi, Chiba, Japan \\ ${ }^{3}$ Tsukuba Research Laboratory, Hitachi Maxell Ltd., 6-20-1 Yawara, Tsukuba-gun, Japan
}

We present results of a time-resolved study of the ultrafast magnetic response of $\mathrm{Gd}_{23.1} \mathrm{Fe}_{71.9} \mathrm{Co}_{5.0}$ MAMMOS structures under conditions near actual read/write temperatures. An all-optical pump and probe method was used in which an intense (pump) light beam excited a medium due to ultrafast laser heating and a less intense (probe) beam monitored this photo-excited state through the magneto-optical Kerr effect. Our experiment clearly demonstrates that the photo-excitation effectively excites coherent spin waves in the magnetic material. Precession frequencies of several $\mathrm{GHz}$ and relaxation times in the nanosecond range were observed.

Key words: MAMMOS, magnetization precession, thermo-magnetic writing

\section{Introduction}

The speed limits for thermomagnetic writing are of vital importance for magneto-optical recording, which became one of the most important technologies for removable storage media. In particular, the speed of the thermally assisted copying within magnetically amplified magneto-optical systems (MAMMOS) is of high technological interest ${ }^{1}$. Conventional pure magnetic recording schemes have a serious and unavoidable problem known as the ferromagnetic resonance (FMR) limit. Nevertheless, laser pulse writing was shown to lead to a potentially much faster process of magnetization reversal, of the order of a few picoseconds ${ }^{2,3)}$. These experiments demonstrated the intrinsic capability of ultrafast high-speed rewritable storage under the thermomagnetic scheme.

In this paper, we describe the results of a time-resolved study of the ultrafast magnetic response of $\mathrm{Gd}_{23.1} \mathrm{Fe}_{71.9} \mathrm{Co}_{5.0}$ MAMMOS structures under the condition near the actual read/write temperature. For the study we used an all-optical pump and probe method in which an intense (pump) light beam excited a medium due to ultrafast laser heating and a less intense (probe) beam monitored this photo-excited state through the magneto-optical Kerr effect ${ }^{4}$. In this way, response times and damping can directly be measured in the time domain with femtosecond resolution. Then, the damping factor was determined from the directly fitting of the measured result with model calculation, which is important issue remained in previous report ${ }^{4}$.

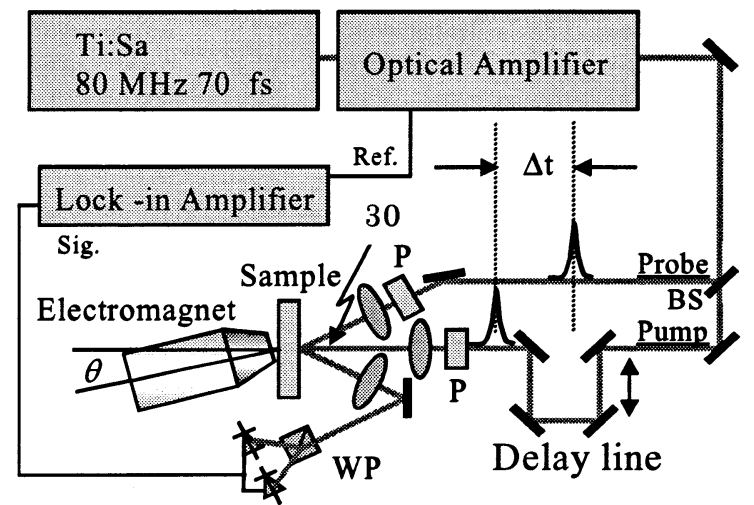

Fig. 1 Schematic diagram of measuring system.

The spatial resolution of this technique is limited only by the wave character of light, in principle allowing for the study of sub- $\mu \mathrm{m}$ structures. This method is also useful for the characterization of the new types of fast switching magnetic devices such as the magnetic random access memory (MRAM) ${ }^{5,6)}$. In contrast, the FMR method, which is widely used for the dynamic characterization of magnetic materials, is normally applied as a nonlocal technique only. Since FMR measurements are taken in the frequency domain, information on damping can only be indirectly obtained from the broadening of the absorption lines.

\section{Method}

The experimental setup is shown schematically in Fig. 1. A commercial Ti:sapphire regenerative amplified laser system provided 150 fs laser pulses with a center wavelength of $800 \mathrm{~nm}$ and a repetition rate of $1 \mathrm{kHz}$. The laser beam was split into pump and probe beams by a beam splitter. After passing a delay line, the linearly polarized pump beam was focused onto the sample surface at normal incidence. A spot diameter of approximately $100 \mu \mathrm{m}$ led to an incident pump fluence of $50 \mathrm{~mJ} / \mathrm{cm}^{2}$. The sample was placed in front of a magnet pole. Using an intense laser pump-pulse, the material is almost instantaneously heated. The effect on the magnetization is measured by a much weaker, time-delayed probe-pulse using the magneto-optical Kerr 


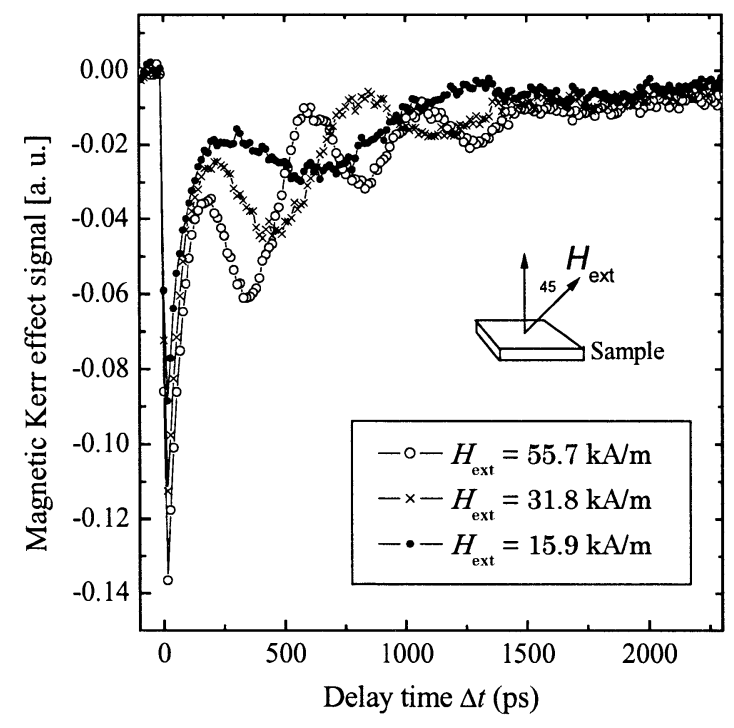

Fig. 2 Typical measurement on a $20 \mathrm{~nm} \mathrm{GdFeCo}$ film displaying the magnetization for various external fields (15.9, $31.8,55.7 \mathrm{kA} / \mathrm{m})$. The magnetization is measured by the polarization state of the reflected probe pulse. When the pump-pulse heats the material at delay time $\Delta t=0$, a sharp decrease in $M$ is observed. A clear damped oscillation is observed, with a frequency that increases with external applied field.

effect. The probe beam was polarized with a Glan-Laser polarizer, and then focused onto the sample at an angle of incidence of $30^{\circ}$. The resulting spot diameter of the probe was about $25 \%$ of that of the pump beam, which guaranteed a homogeneously excited probing region. After reflection from the sample and collimation, the Kerr rotation $\theta$ was detected by using a balanced photodiode scheme.

By varying the time delay between pump and probe, the magnetization can be measured as a function of the time delay after excitation. An external magnetic field was applied with an electromagnet under various angles with respect to the sample normal. The bias temperature $T_{\text {bias }}$ of the sample was controlled by a ceramic heater. Throughout this study $T_{\text {bias }}$ was constant $(403 \mathrm{~K})$.

The sample was a MAMMOS read-out layer, consisting of a multilayer structure. A $100 \mathrm{~nm}$ thick layer of AlTi on top of the glass substrate served as a heat sink and enhanced the reflectivity of the sample. The sample used was a $\mathrm{SiN}(60 \mathrm{~nm}) / \mathrm{Gd}_{23} \mathrm{Fe}_{72} \mathrm{Co}_{5}(20 \mathrm{~nm}) / \mathrm{SiN}(5$ $\mathrm{nm}) / \operatorname{AlTi}(100 \mathrm{~nm}) /$ glass grown by magnetron sputtering. Coercivity $H \mathrm{c}$ and magnetization $M \mathrm{~s}$ were $13.6 \mathrm{kA} / \mathrm{m}$ (171 Oe) and $83 \mathrm{emu} / \mathrm{cm}^{3}$ respectively at $403 \mathrm{~K}$. The ferrimagnetism of $\mathrm{GdFeCo}$ results from antiferromagnetic coupling of the ferromagnetic rear earth (RE) and transition metal (TM) subsystems. For our sample, Curie temperature and compensation temperature were $\sim 518$ and $\sim 260 \mathrm{~K}$, respectively. Note that only the magnetization of

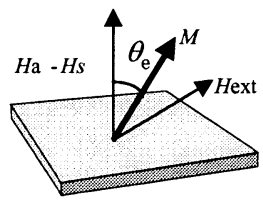

I: The magnetization is canted as equilibrium direction $\theta_{\mathrm{e}}$

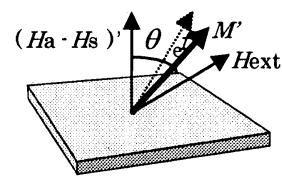

II: Anisotropy and magnetization change due to heating by the pump-pulse. Change of the equilibrium orientation from $\theta_{\mathrm{e}}$ to $\theta_{e}^{\prime}$, trigger an initial

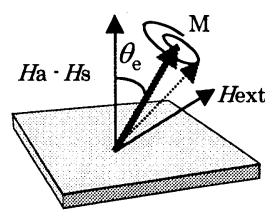
precession.

\section{III: Original equilibrium angle $\theta_{\mathrm{e}}$ is rapidly restored with precessional motion.}

Fig. 3 Schematic diagram of the excitation process. I: equilibrium direction; II: anisotropy and $M$ change due to heating; III: precession starts. $\boldsymbol{H}_{\text {ext }}$ is the external applied field; $\boldsymbol{H}_{\mathrm{a}}$ and $\boldsymbol{H}_{\mathrm{s}}$ represent the effective perpendicular- and shape- anisotropy fields of the film respectively.

the TM subsystem is probed by the linear Kerr-effect at $800 \mathrm{~nm}$.

\section{Results and discussions}

Typical measurements on a $20 \mathrm{~nm}$ thick GdFeCo film displaying a magnetization $\boldsymbol{M}$ for various external fields (15.9 (200 Oe), 31.8 (400 Oe), $55.7 \mathrm{kA} / \mathrm{m}$ (700Oe) ) are shown in Fig. 2. When the pump-pulse heats the material at delay time $\Delta t=0$, a sharp decrease in $M$ is observed. This effect is caused by a sudden change in magnitude of the temperature dependent magnetization. The subsequent recovery of $M$ on a time scale of about one hundred ps is due to rapid heat diffusion into the substrate. In addition, a clear damped oscillation is observed, with a frequency that increases with the external applied field.

\subsection{Excitation process}

Fig. 3 shows a schematic diagram of the excitation process. By applying an appropriate external field $H_{\text {ext }}$ the magnetization is canted. The canting angle $\theta_{\mathrm{e}}$ is determined by a balance between the external field and the anisotropy field of the film, including the shape anisotropy. Upon sudden heating by the pump-pulse (i) the magnetization decreases, leading to a change of the shape anisotropy, and (ii) the magnetocrystalline anisotropy changes as well ${ }^{4)}$. Altogether, this results in a change of the equilibrium orientation from $\theta_{\mathrm{e}}$ to $\theta_{e}^{\prime}$, triggering an initial precession of the magnetization around its new equilibrium orientation. Heat diffusion into the film quickly removes the excess heat and, after some hundreds of ps, the original equilibrium angle is restored. However, at this point the magnetization is still not in equilibrium due to its initial displacement, and will 


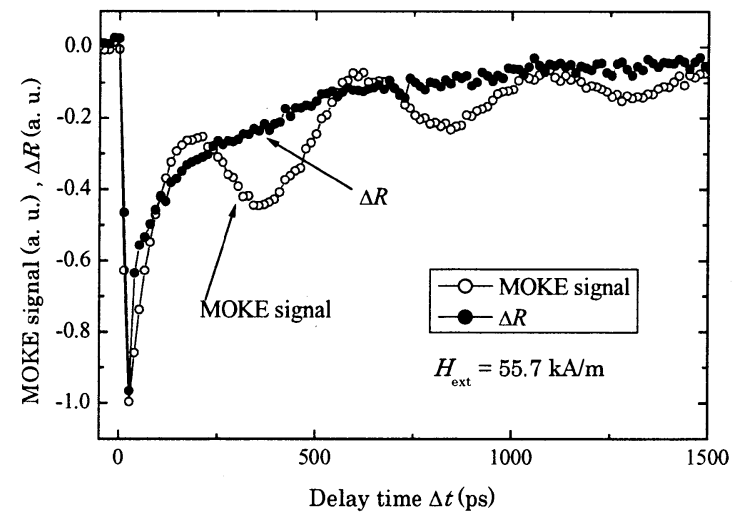

(a) Long time scale

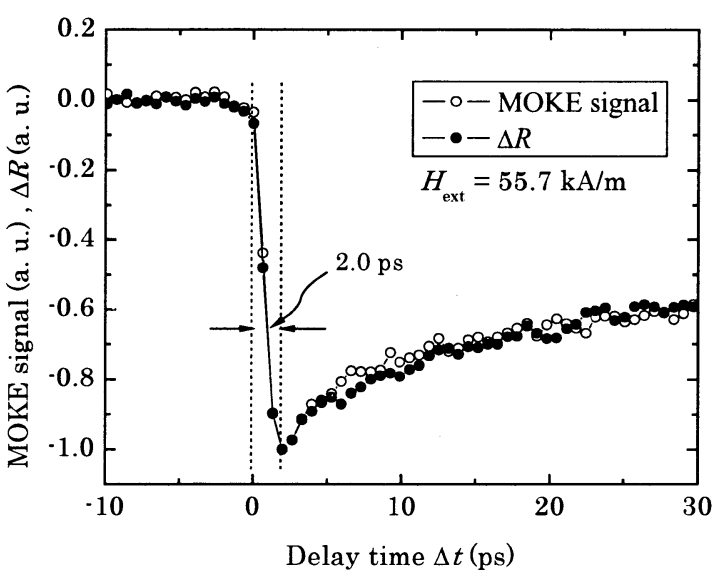

(b) Short time scale

Fig. 4 Magnetization of the $\mathrm{GdFeCo}$ (open circle) and simultaneously measured changes of reflectivity $(\Delta R)$ monitoring transient electron temperatures (solid circle), as a function of pump probe delay at (a) long time scale, (b) short time scale. After the excitation, the electron temperature reaches its Indaximum value within a few ps and then decreases with a time constant of $t_{\mathrm{tc}}=145 \mathrm{ps}$. In this period, changes of both magnetic anisotropy and magnetization take place. This is equivalent to a short effective magnetic field applied to the sample.

continue to precess for a few nanoseconds.

In order to measure the changes of the magneto-optical response, which were exclusively caused by transient electron- and lattice temperature, we applied a permanent saturation field of $55.7 \mathrm{kA} / \mathrm{m}$. Corresponding results are shown in Fig. 4. The magnetization dynamics is compared with the simultaneously measured changes of reflectivity that monitor the time evolution of the electron temperature. After the excitation the electron temperature reached its maximum value within a few ps and then decreased with a time constant of $t_{\mathrm{tc}}=145 \mathrm{ps}$. In this period, the changes of both the magnetic anisotropy and the magnetization took place. This is equivalent to a short effective magnetic field applied to the sample ${ }^{7)}$.

Additionally, it follows that the exact excitation

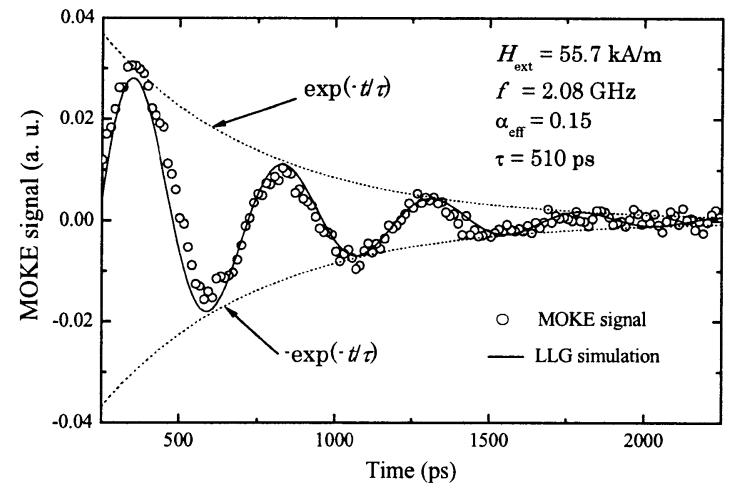

Fig. 5 Magnetization response as a function of time for bias field $15.9 \mathrm{kA} / \mathrm{m}$. It determined by eliminate non-oscillate term caused thermal effect from measured result shown in Fig. 4. The experimental curve is very well fitted by the Landau-Lifshitz-Gilbert equation (solid line) with a effective damping parameter $\alpha_{\text {eff }}=0.15$.

mechanism is not of importance for the result; i.e., the short time excitation determines the amplitude and phase of the final precession, but not its precession frequency and damping.

\subsection{Precession dynamics}

The spin dynamics of the ferrimagnetic GdFeCo system can be well described by a phenomenological description offered by Landau, Lifshitz and Gilbert:

$\mathrm{d} \boldsymbol{M} / \mathrm{d} t=\gamma_{\text {eff }}(\boldsymbol{M} \times \boldsymbol{H})-\alpha_{\text {eff }} \boldsymbol{M}(\boldsymbol{M} \times \mathrm{d} \boldsymbol{M} / \mathrm{d} t)$

where $\gamma_{\text {eff }}$ is the effective gyromagnetic ratio. The value of $\gamma_{\text {eff }}$ is given by $-g_{\text {eff }} \mu_{\mathrm{B}} / \hbar$ under the assumption of the spins of all sublattices are strongly coupled and act on the same precession, where $g_{\text {eff }}$ and $\mu_{\mathrm{B}}$ are the effective spectroscopic splitting factor and Bohr magneton, respectively. The effective value of Gilbert damping of the system is represented by $\alpha_{\text {eff. }}$ In Eq. (1) $\boldsymbol{H}$ denotes the total effective field in the system:

$\boldsymbol{H}=\boldsymbol{H}_{\mathrm{ext}}+\boldsymbol{H}_{\mathrm{a}}+\boldsymbol{H}_{\mathrm{s}}$

where $\boldsymbol{H}_{\text {ext }}$ is the external applied field; $\boldsymbol{H}_{\mathrm{a}}$ and $\boldsymbol{H}_{\mathrm{s}}$ represent the effective perpendicular- and shape anisotropy fields of the film respectively.

The $\alpha_{\text {eff }}$ could be derived by directly fitting the time dependence of the time domain MOKE measurement with Eq. (1). Fig. 5 shows the magnetization response as a function of time for a bias field of $15.9 \mathrm{kA} / \mathrm{m}$. It was determined by eliminating the non-oscillating term caused by the thermal effect of the measurement, shown in Fig. 4. The experimental curve is very well fitted by the Landau-Lifshitz-Gilbert equation (solid line in Fig. 5) with $\alpha_{\text {eff }}=0.15$. For this fit a precession frequency $f=$ $2.08 \mathrm{GHz}$ and a decay time of $\tau=1 / 2 \pi f \alpha_{\mathrm{eff}}=510 \mathrm{ps}$ have been used. The precession frequency was determined by non-linear fitting of the measured result with a damped 
harmonic equation.

In general the precession frequency $f$ is given by the determinant of the coupled system of differential equations. The exact calculation of $f$ is rather complicated since the time dependent effective field has to be included. For large external applied fields, however, its value is given in good approximation by assuming that $\boldsymbol{M}$ is constant and equal to the saturation magnetization $\boldsymbol{M}_{\mathrm{s}}$ after excitation. It is then straightforward to obtain:

$$
f=\gamma_{e f f} / 2 \pi\left(1+\alpha_{e f f}^{2}\right) \cdot \sqrt{\left(H_{e x t} \cos \theta+H_{a}+H_{s}\right)^{2}-\left(H_{e x t} \sin \theta\right)^{2}}
$$

where $\theta$ represents the angle between external field and the normal to the film plane. A change of this effective field in the thin film will influence the precession frequency. If the angular momentum is due to the spin only, $\mathrm{g}_{\text {eff }}=2$. If the orbital angular momentum also contributes it is less than 2 . In transition metal ions of Fe and $\mathrm{Co}$, the orbital angular momentum are quenched, so $\mathrm{g}_{\text {eff }}=2$. The 4-f electrons in the rear earth ions are so deeply buried in the inner shells that external influences are screened by the outer shells. Consequently these ions preserve there free-atom characteristics. Therefore, $\mathrm{g}_{\text {eff }}=$ 2 for Gd, since it has no orbital momentum. Thus it is expected that the effective spectroscopic splitting factor $\mathrm{g}_{\text {eff }}$ is dominated by the spin part of angular momentum in the GdFeCo alloy case.

Fig. 6 shows the external field dependence of the precession frequency. The precession frequencies were determined by non-linear fitting of the measured results in Fig. 2 with a damped harmonic function. Eq. (3) was then used to fit the determined values as shown in Fig. 5 (solid line). As shown, the external field is added to the effective internal field and the vector sum will increase the precession frequency. In our fits to the measured precessions, we took the previous result $\alpha_{\text {eff }}=0.15$. Under the assumption $\mathrm{g}_{\text {eff }}=\mathrm{g}_{\mathrm{RE}}=\mathrm{g}_{\mathrm{TM}}$, the measurement is well described with $\mathrm{g}_{\mathrm{eff}}=1.85$. This result is in reasonable agreement with FMR esults measured in a Gd- TM amorphous alloy system (i.e. $\left.\mathrm{GdFe}_{2}: \mathrm{g}_{\text {eff }} \sim 1.75\right)^{8}$.

Thus this all-optical method is capable of reproducing the magnetization dynamics after the system has been driven out of the equilibrium by an optical excitation that is equivalent to a hundred picoseconds magnetic field pulse.

\section{Conclusion}

By an all-optical pump and probe method we demonstrated that the photo-excitation of a thin film of $\mathrm{GdFeCo}$ could effectively excite coherent spin waves in the magnetic material. An intense (pump) optical beam excites the medium due to an ultrafast laser-induced heating and a less intense (probe) beam monitores this photo-excited state through the magneto-optical Kerr effect. Time-domain measurements on the consequently excited precession give information on the magnetic anisotropy, switching and damping phenomena. The

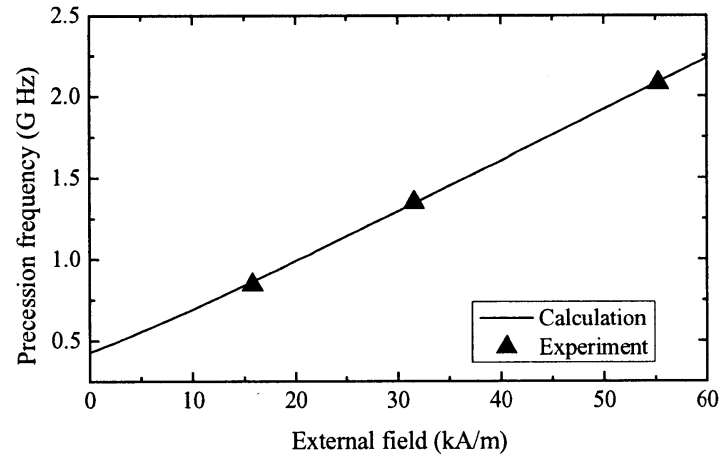

Fig. 6 External field dependence of the precession frequency. The solid line shows the fit to Eq. (3). The precession frequencies were determined by nonlinear fitting of the measured results in Fig. 2 with a damped harmonic function. As shown, the external field is added to the effective internal field, and the vector sum increases the precession frequency. In our fits to the measured precessions, we took $\alpha_{\text {eff }}=0.15$ determined from the fitting shown in Fig. 5. Under the assumption $\mathrm{g}_{\text {eff }}=$ $\mathrm{g}_{\mathrm{RE}}=\mathrm{g}_{\mathrm{TM}}$, the measurement is well described with $\mathrm{g}_{\mathrm{eff}}=1.85$.

ultrafast magnetic response of $\mathrm{Gd}_{23.1} \mathrm{Fe}_{71.9} \mathrm{Co}_{5.0}$ MAMMOS structures was measured under near actual $\mathrm{read} /$ write temperature condition. Precession frequencies of several $\mathrm{GHz}$ and relaxation times in the nanosecond range were observed under various external magnetic fields. The experimental data are in good agreement with our calculations on the basis of the Landau-Lifshitz-Gilbert equation.

Acknowledgment This work is partially supported by a Grant-in-Aid for Scientific Research of the Ministry of Education and economy in Japan No. 15760226, the European Union IST project SPINOSA (Contract No. IST-2001-33334) and the Dutch organization for Fundamental Research on Matter (FOM).

\section{References}

1) H. Awano, S. Imai, M. Sekine, M. Tani, N. Ohta, K. Mitani, N. Takagi, H. Noguchi, and M. Kume: IEEE Trans. Magn. 362261 (2000).

2) J. Hohlfeld, T. Gerrits, M. Bilderbeek, H. Awano, N. Ohta, and T. Rasing, Trans. Magn. Soc. Jpn, 25202 (2001).

3) J. Hohlfeld, Th. Gerrits, M. Bilderbeek, Th. Rasing, H. Awano, and N. Ohta, Phys. Rev. B 65012413 (2002).

4) A. Tsukamoto, K. Nakagawa, A. Itoh, A. Kirilyuk, T. Rasing and H. Awano: J. Magn. Soc. Jpn. 28318 (2004) (Japanese)

5) M. van Kampen, C. Jozsa, J. T. Kohlhepp, P. LeClair, L. Lagae, W. J. M. de Jonge, and B. Koopmans: Phys. Rev. Lett. 88227201 (2002).

6) Th. Gerrits, H. A. M. van den Berg, J. Hohlfeld, L. Bar, and Th. Rasing: Nature 418509 (2002).

7) T. J. Silva, P. Kabos, and M. R. Putfall, Appl. Phys. Lett. 81 2205 (2002)

8) P. Lubitz, J. Schelleng, C. Vittoria: J. Magn. Magn. Mater. 29178 (1975)

Received April 16, 2004; Accepted July 27, 2004. 